of $0.6 \mathrm{ml}$ of $105 \mathrm{mM} \mathrm{K}$-phosphate buffer ( $\mathrm{pH} 8.0$ ), containing $0.1 \%$ bovine serum albumin, $0.5 \mathrm{mM} \mathrm{ATP}, 0.5 \mathrm{mM}$ fructose1,6-bisphosphate, $0.5 \mathrm{mM} \mathrm{MgCl}$ and $0.15 \mathrm{mM} \mathrm{CaCl}_{2}$ for $60 \mathrm{~min}$ at room temperature. After the addition of $0.1 \mathrm{ml}$ of sensitized sheep erythrocytes $\left[4 \cdot 10^{6}\right.$ Ery preincubated with $0.005 \mathrm{ml}$ amboceptor (Behringwerke) for $60 \mathrm{~min}$ at $20 \mathrm{Cl}$, incubation was continued for another $60 \mathrm{~min}$ at $37 \mathrm{C}$. Unlysed erythrocytes were removed by centrifugation and lysis was measured at $416 \mathrm{~nm}$ in the supernatants after addition of $0.1 \mu$ mole $\mathrm{KCN}$.

\section{Results}

Immunoprecipitation of PFK. Figure 1 shows the variation in the amount of anti-rabbit muscle PFK serum required to precipitate PFK from different mammalian muscles. The antibodies precipitate ${ }^{125}$ I-labelled PFK in the same ratio as unlabelled PFK. The precipitation of PFK from various tissues by antiserum against rabbit muscle PFK offered the possibility for a convenient determination of unknown concentrations of PFK protein. Amounts of $2-6 \mu \mathrm{g}$ of rabbit muscle PFK were accurately estimated by a simple radioimmunoassay. The sensitivity of the technique increases inversely proportional to the affinity of the labelled PFK indicator for the antiserum. The requirement of freshly prepared $\left[{ }^{125} \mathrm{I}\right]-\mathrm{PFK}$ is the major disadvantage of this method.

Complement Fixation Test. Complement fixation tests were used for the determination of 3-30 ng of PFK with good reproducibility. The test does not differentiate between active and inactive PFK. The method was successfully applied to the

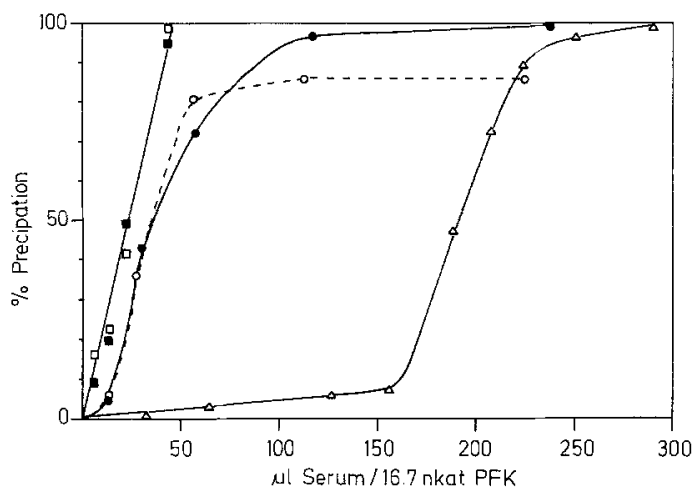

Fig. 1. Precipitation of PFK from different species by antirabbit muscle PFK serum. $\square$ Rabbit muscle PFK; ${ }^{125}$ Irabbit muscle PFK; O pig muscle PFK; beef muscle PFK; $\triangle$ human muscle PFK

determination of the specific activity of PFK in human muscle extracts.

The study was supported by the Deutsche Forschungsgemeinschaft and the Fonds der Chemischen Industrie.

\section{References}

1. Bartholomé-Dönnicke, M., Hofer, H. W.: Biochim. Biophys. Acta 397, $347-354$ (1975)

2. Hofer, H. W., Pette, D.: Z. Physiol. Chem. 349, 995- 1012 (1968)

\title{
6.4 .
}

Fresenius Z. Anal. Chem. 290, 129-130 (1978) - C by Springer-Verlag 1978

\section{Beiträge zur rechnerunterstützten Diagnostik II. Gruppierung biochemischer Schilddriisenparameter ohne Beriicksichtigung der klinischen Diagnose}

P. Sandel, W. Vogt, S. Broda, D. Jüngst und $M$. Knedel

Institut für Klinische Chemie und Medizinische Klinik II am Klinikum Großhadern der Universität München, Postfach 702260,8000 München 70

\section{Contributions to Computer Assisted Diagnostics: \\ II. Grouping of Biochemical Thyroid Parameters without Considering Clinical Diagnosis}

Key words: Untersuchung der Schilddrüse; Gruppierung der Parameter

Der Stellenwert der Laboratoriumsuntersuchungen in der Schilddrüsendiagnostik allgemein ist unbestritten. Bei der Frage jedoch, welche Bewertung den einzelnen Parametern in der Diagnostik der Schilddrüsenerkrankungen zukommt, gehen die Ansichten bereits weit auseinander. Grund für solche Differenzen ist die Tatsache, daß die Beurteilung der Laborwerte bisher weitgehend empirisch erfolgte.

Dank leistungsfähiger Computer ist es nun aber möglich, die empirische Vorgehensweise durch eine analytische $\mathrm{zu}$ ersetzen, und so rücken mehrdimensionale statistische Analysen von Laborwerten immer stärker in den Vordergrund des Interesses der labordiagnostischen Forschung. Es sind dies vor allem Clusteranalysen und Diskriminanzanalysen.

Ziel unserer Untersuchungen war es, im Rahmen der Schilddrüsendiagnostik zur Klärung folgender Fragen beizutragen:

Welche Gruppierungen lassen sich in den Laborwerten finden?

Sind und wie sind die gefundenen Gruppen pathophysiologisch $\mathrm{zu}$ interpretieren?

Mit welcher Strategie - parallel bzw. seriell - sind die Laboranalysen am zweckmäßigsten durchzuführen?

Mit welchem Unsicherheitsfaktor ist die Aussage der verschiedenen Laboranalysen behaftet?

Die Daten, mit denen die Untersuchungen durchgeführt wurden, stammen aus der endokrinologischen Ambulanz des Klinikums Großhadern. Von insgesamt 322 Patienten mit Verdacht auf Schilddrüsenerkrankung wurden die anamnestischen Daten, die Laborwerte, die klinischen, ohne Kennt- 


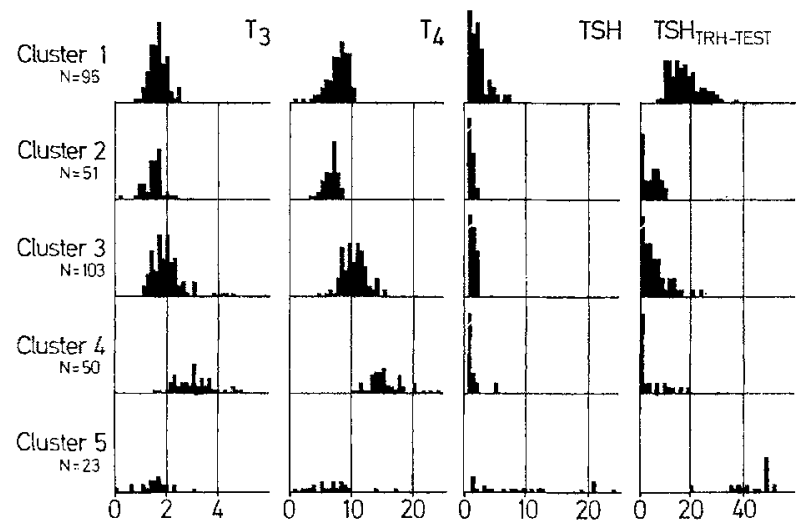

Abb.1. Verteilungen von $\mathrm{T}_{3}, \mathrm{~T}_{4}$, TSH und TSH nach TRHTest in den fünf entsprechend dem Ergebnis der Clusteranaly. se gewählten Gruppen

nis der Laborwerte gestellten Diagnosen und die Enddiagnosen erfaßt. Die im folgenden verwendeten Laborwerte waren $\mathrm{T}_{3}, \mathrm{~T}_{4}$, TSH und TSH nach TRH-Test.
Um festzustellen, welche Gruppierungen in den Laborwerten stecken, haben wir eine Clusteranalyse durchgeführt. Entsprechend dem Ergebnis der Clusteranalyse haben wir eine Aufteilung in 5 Gruppen gewählt. Die Verteilungen der $\mathrm{T}_{3^{-}}, \mathrm{T}_{4^{-}}$und TSH-Werte sind für die 5 Gruppen getrennt in Abbildung 1 dargestellt. Gruppe 1 enthält im wesentlichen die Patienten mit unveränderter Schilddrüsenfunktion. Gruppe 2 ist vor allem als Gruppe der Patienten mit , latenter Hyperthyreose" anzusehen. Gruppe 3 ist wohl auch als Gruppe der latenten Hyperthyreosen anzusehen; hier besteht gleichzeitig aber eine leichte $\mathrm{T}_{4}$-Erhöhung. In Gruppe 4 sind eindeutig die hyperthyreoten Patienten zusammengefaßt und Gruppe 5 schließlich enthält im wesentlichen die Patienten mit hypothyreoter Stoffwechsellage.

Die labordiagnostische Bedeutung dieser Gruppen ergibt sich aus ihrer jeweiligen klinisch-therapeutischen Relevanz, die ebenfalls Gegenstand dieser Untersuchungen war. Die Definierung von labordiagnostischen Gruppen anhand ihrer klinisch-therapeutischen Relevanz ist Voraussetzung für die Festlegung der Strategie, wie - parallel bzw. seriell - die Laboranalysen zweckmäßigerweise durchzuführen sind. Ebenso kann die Frage nach den predictive values einer Laboranalyse erst beantwortet werden, wenn die Definitionen der labordiagnostischen Gruppen festliegen. Denn erst wenn festliegt, welche Labordiagnosen gegeneinander abzugrenzen sind, kann die Güte der Abgrenzung angegeben werden.

6.5 .

Fresenius Z. Anal. Chem. 290,130-131 (1978) - (C) by Springer-Verlag 1978

\section{Ein Thermistordetektor zur Bestimmung von Substraten an Träger-gebundenen Enzymen in Durchfluß-Systemen}

\section{G. Krisam}

Institut für Chemie Weihenstephan der TU München, D-8050 Freising-Weihenstephan

\section{A Thermistor Detector for Substrate Determination with Immobilized Enzymes in Flow Systems}

Key words: Substratbest. in Biolog. Flüssigkeiten; Thermometrie; Thermistordetektor, Durchflußsystem

Thermometrische Substratbestimmungen lassen sich in Durchflußsystemen durchführen, wenn die Entwicklung der Reaktionswärme örtlich fixiert ist; dies ist der Fall bei der Katalyse der Reaktion durch einen heterogenen Katalysator. Heterogene Biokatalysatoren sind Träger-gebundene Enzyme, und die Kombination von Enzymreaktoren mit Thermistoren führt zu spezifischen empfindlichen Substratdetektoren (Enzymthermistoren) für Durchflußsysteme [3]. In dem von uns beschriebenen System [1] wird die Temperaturdifferenz zwischen Anfang und Ende des Enzymreaktors mit zwei Thermistoren über eine Wheatstonesche Brückenschaltung mit nachgeschaltetem Operationsverstärker gemessen; die Anzeige erfolgt über einen Schreiber. Meßbrücke und Detektor werden elektronisch thermostatisiert.
Reaktoren auf der Basis von Enzymgelen erwiesen sich als ungeeignet für Substratbestimmungen in biologischen Flüssigkeiten wie Serum und Urin, da nicht-reproduzierbare Wärmeeffekte auftraten, die wahrscheinlich durch Adsorptions- und Desorptionsvorgänge verursacht wurden; schließlich verstopften auch die Reaktoren. Daher wurden die Enzyme kovalent an die Innenwand von Nylonschläuchen gebunden [2]. In Meßzellen mit solchen Schläuchen traten bei Messungen in biologischen Flüssigkeiten nur noch geringe, kompensierbare Nebenwärmen auf. Inaktivierungen des Katalysators wurden lediglich durch nicht-entrahmte Milch hervorgerufen (Fettablagerung). Mit so gebundener Urease konnte Harnstoff in $30 \mu 1$-Proben von Harn oder Serum in einem Bereich von $0,1-1,5 \mu \mathrm{Mol}$ (Fehler $\pm 0,05 \mu \mathrm{Mol}$ ) bestimmt werden. Ein Enzymreaktor mit dem gekoppelten Enzymsystem Glucose-Oxidase/Katalase konnte zur Glucosebestimmung in nativem Serum und Urin in einem Bereich von $0,05-0,5 \mu \mathrm{mol}$ bei einem Fehler von $0,03 \mu \mathrm{mol}$ eingesetzt werden. Der große relative Fehler im unteren Bereich ist durch konvektionsbedingte Temperaturschwankungen verursacht, an deren Beseitigung zur Zeit gearbeitet wird. Im oberen Bereich der Eichkurve entsprechen Empfindlichkeit und Genauigkeit nahezu den Werten optischer Verfahren.

Als sehr vorteilhaft zur Steigerung der Wärmeausbeute beim Umsatz eines Substrates erwies sich die Kopplung von Enzymreaktionen. So konnte durch die Verbindung der Reaktion $\mathrm{O}_{2}$-abhängiger Flavoproteide mit der Katalasereaktion, z. $\bar{B}$. bei der Harnsäurebestimmung mit Uricase, eine Steigerung der Empfindlichkeit auf das Doppelte erzielt werden. Schließlich wurden Systeme mit drei gemeinsam 